\title{
Avaliação da eficácia do tratamento de bruxismo com placa miorrelaxante e aplicação de tens por meio de análise eletromiográfica
}

\author{
Electromyographic analysis evaluation of the bruxism treatment \\ with oclusal splint therapy and TENS efficacy \\ Evaluación de la eficacia del tratamiento de bruxismo con placa miorrelaxante \\ $y$ aplicación de TENS por medio de análisis electromiográfico \\ Jeniffer Roberta BEZERRA ${ }^{1}$ \\ Andréia Maria SILVA ${ }^{2}$ \\ Marcela Filié HADDAD ${ }^{1}$ \\ ${ }^{1}$ Departamento de Odontologia Restauradora, Faculdade de Odontologia, \\ Universidade Federal de Alfenas (UNIFAL-MG), 37.130-000, Alfenas - MG, Brasil \\ ${ }^{2}$ Escola de Enfermagem, Curso de Fisioterapia, \\ Universidade Federal de Alfenas (UNIFAL-MG), 37.130-000, Alfenas - MG, Brasil
}

\begin{abstract}
Resumo
Introdução: Bruxismo é uma desordem funcional multifatorial que acomete diversas faixas etárias, apresentando maior incidência em jovens, caracterizada pelo apertamento e ranger de dentes. Seu tratamento requer múltiplas intervenções para a adequada reabilitação do estado de normalidade funcional do paciente, sendo que a mais comum é a instalação de placas miorrelaxantes; e uma segunda intervenção, menos corriqueiramente utilizada, é a Estimulação Elétrica Nervosa Transcutânea (TENS). Objetivos: Analisar, por meio de eletromiografia, a eficiência do uso de placas miorrelaxantes no período noturno e a aplicação de TENS semanalmente, na melhora das condições de desconforto advindas do bruxismo. Material e Método: Foram selecionados 20 indivíduos portadores de bruxismo. Inicialmente, estes pacientes passaram por análise eletromiográfica. Em seguida, todos receberam placas miorrelaxantes e foram orientados a dormir com as mesmas durante 30 dias. Foram submetidos também à aplicação de TENS uma vez por semana durante este mesmo período. Passados 30 dias de tratamento, foi realizada nova análise eletromiográfica para observação da evolução do tratamento. Resultados: Os resultados encontrados no presente estudo revelaram redução significativa da atividade muscular para o músculo temporal durante o repouso muscular. De modo geral, observou-se redução da atividade eletromiográfica durante a contração para o masseter e aumento para o temporal, porém sem diferença estatística. Conclusão: O tratamento do bruxismo com placa miorrelaxante rígida e TENS promoveu redução da atividade eletromiográfica para o músculo temporal durante o repouso muscular, sugerindo que o tratamento proposto proporciona melhora da sintomatologia do bruxismo.
\end{abstract}

Descritores: Bruxismo; Estimulação Elétrica Nervosa Transcutânea; Eletromiografia.

\begin{abstract}
Introduction: Bruxism is a multifactorial functional disorder that affects different age groups, with higher incidence in youths and young adults, characterized by clenching and grinding of teeth. The treatment requires multiple interventions for proper rehabilitation of the patient's functional normality status. The most common treatment is the use of myorelaxing plaques and as a second intervention, which may be cited but is less routinely used, is the use of TENS (Transcutaneous Electrical Nerve Stimulation). Objectives: Analyse the efficiency of the use of myorelaxing plaques at night and the use of TENS weekly in order to detect, after 30 days of treatment, an improvement of the discomfort conditions caused by bruxism. Matherial and Method: Twenty individuals with bruxism were selected. Initially, the electromyographic analysis was done. Then, all of them received myorelaxing plaques and were told to sleep with them for 30 days. They were also submitted to TENS therapy once a week during this same period. After 30 days of treatment, a new electromyographic analysis was performed to observe the evolution of the treatment. Results: There was a significant reduction of muscle activity to the temporal muscle during muscle rest. In general, there was a reduction of electromyographic activity during contraction for the masseter and increase for the temporal, but without statistical difference. Conclusion: Bruxism treatment with rigid myorelaxing plaques and TENS promoted a reduction of the temporal muscle electromyographic activity during muscle rest, suggesting that the proposed treatment improves the bruxism symptomatology. Descriptors: Bruxism; Transcutaneous Electric Nerve Stimulation; Electromyography.
\end{abstract}

\section{Resumen}

Introducción: El bruxismo es un desorden funcional multifactorial que acomete diversas edades, presentando mayor incidencia en jóvenes, caracterizada por el aprieto y el crujir de dientes. Su tratamiento requiere múltiples intervenciones para la adecuada rehabilitación del estado de normalidad funcional del paciente, siendo que la más común es la instalación de placas miorrelaxantes; Y una segunda intervención, menos habitualmente utilizada, es la Estimulación Eléctrica Nerviosa Transcutánea (TENS). Objetivos: Analizar, por medio de electromiografía, la eficiencia del uso de placas miorrelaxantes en el período nocturno y la aplicación de TENS semanalmente, en la mejora de las condiciones de incomodidad derivadas del bruxismo. Material y Método: Fueron seleccionados 20 individuos portadores de bruxismo. Inicialmente, estos pacientes pasaron por análisis electromiográfico. A continuación, todos recibieron placas miorrelaxantes y fueron orientados a dormir con las mismas durante 30 días. Se sometieron también a la aplicación de TENS una vez por semana durante este mismo período. Después de 30 días de tratamiento, se realizó un nuevo análisis electromiográfico para observar la evolución del tratamiento. Resultados: Los resultados encontrados en el presente estudio revelaron una reducción significativa de la actividad muscular para el músculo temporal durante el reposo muscular. En general, se observó reducción de la actividad electromiográfica durante la contracción para el masseter y aumento para el temporal, pero sin diferencia estadística. Conclusión: El tratamiento del bruxismo con placa miorrelaxante rígida y TENS promovió reducción de la actividad electromiográfica para el músculo temporal durante el reposo muscular, sugiriendo que el tratamiento propuesto proporciona mejoría de la sintomatología del bruxismo.

Descriptores: Bruxismo; Estimulación Eléctrica Transcutánea del Nervio; Electromiografía.

\section{INTRODUÇÃO}

$\mathrm{O}$ ranger ou o apertar dos dentes pode ser um ato realizado de forma consciente ou inconsciente, caracterizado pelo contato não funcional dos mesmos. Não é uma doença, mas quando exacerbado pode levar a um desequilíbrio fisiopatológico do sistema estomatognático, designado bruxismo $^{1}$.

A etiologia é multifatorial, ou seja, pode estar relacionada a fatores locais, sistêmicos, psicológicos e 
hereditários. A literatura define sua origem como multicausal, complexa e de difícil detecção. Os sinais e sintomas relatados pelo paciente são os principais métodos para identificação do transtorno, além de uma boa avaliação clínica analisando, por exemplo, a sensibilidade à palpação e rigidez muscular do masseter e do temporal ${ }^{2}$.

Apesar do tratamento do bruxismo ser complexo, alguns recursos como a placa de mordida ajudam a eliminar alguns dos danos causados pelo mesmo. Outros tipos de auxílios como terapia de massa e equilíbrio da oclusão e Estimulação Elétrica Nervosa Transcutânea (TENS) também são utilizados na tentativa de se reduzir a dor e a tensão muscular $^{3,4}$.

Como meios de acompanhamento do tratamento podem ser citados exames clínicos, onde os profissionais usam questionários com a finalidade de avaliarem a melhora da sintomatologia apresentada pelo paciente; e a eletromiografia, que consiste em um método apropriado para obter medidas fiéis de freqüência, intensidade e duração da contração muscular ${ }^{2,5}$.

Assim, o propósito deste estudo foi realizar uma avaliação do tratamento do bruxismo com placa miorrelaxante e aplicação de TENS por meio da análise da atividade muscular por eletromiografia.

\section{MATERIAL E MÉTODO}

Inicialmente, este projeto foi submetido ao Comitê de ética em Pesquisa da Universidade Federal de Alfenas (UNIFAL-MG) e obteve parecer favorável (número do parecer de aprovação: 1.291.355; CAAE: 39143214.6.0000.5142, aprovado em 22/10/2015). A pesquisa foi conduzida seguindo os princípios éticos estabelecidos na declaração de Helsinque e da resolução 466/2012. A presente pesquisa foi realizada na clínica e laboratório de prótese, ambos pertencentes ao departamento de Odontologia Restauradora da UNIFAL-MG.

A amostra foi constituída por 20 indivíduos, com idade entre 20 e 50 anos, de ambos os sexos. Estes foram selecionados baseados na aplicação do Eixo I / Formulário de Exame do questionário RDC/TMD, com questões a respeito da presença de dor na face; e em exames clínicos, sendo que aqueles que apresentaram desgaste dentário, principal sintoma do bruxismo excêntrico, foram convidados a participar da pesquisa.

Dentre estes indivíduos selecionados, foram incluídos na presente pesquisa aqueles que apresentavam boas condições de saúde geral, ausência de lesões intraorais e cutâneas, hipertonicidade dos músculos elevadores da mandíbula, desgastes dentários para-funcionais. Indivíduos que apresentaram com alguma debilidade sistêmica, lesões intraorais, que estavam sob tratamento com medicação miorrelaxante, portadores de marca-passo cardíacos ou cerebrais e grávidas foram excluídos da pesquisa, uma vez que esta não engloba variações da normalidade fisiológica ${ }^{2,4}$.

Aqueles que se enquadraram e concordaram em participar assinaram um termo de consentimento livre e esclarecido para a pesquisa, podendo retirar o consentimento a qualquer momento sem nenhuma forma de punição.

Inicialmente, foi feita eletromiografia para registro da atividade muscular no estágio inicial (previamente ao tratamento).

\section{- Eletromiografia:}

A eletromiografia foi realizada utilizando-se um eletromiógrafo contendo filtro analógico ativo Butterworth, passa-faixa; banda passante programada para 20 a $500 \mathrm{~Hz}$; frequência de amostragem com ajuste de ganho até $2000 \mathrm{~Hz}$, tipo de coleta simples com tamanho de janela de 5 segundos. Os eletrodos de referência foram os de formato circular para diminuir o ruído durante a aquisição do sinal eletromiográfico e os eletrodos de superfície bipolares passivos pré-geleificados, $\mathrm{Ag} / \mathrm{AgCl}$ Eletrodo Cardiológico (marca registrada), ambos descartáveis. O eletromiógrafo foi conectado a um computador notebook contendo um software de registro de análise.

Para a coleta do sinal eletromiográfico, a área de fixação dos eletrodos foi limpa com álcool $70 \%$ para favorecer a fixação dos mesmos e impedir a impedância da pele. Os voluntários avaliados estiveram sentados, eretos, com os pés apoiados sobre o solo, os braços relaxados, costas bem apoiadas e plano de Frankfurt paralelo ao solo ${ }^{2}$.

Os eletrodos ativos bipolares superficiais foram fixados nos músculos: masseter (porção superior) e temporal (porção anterior) com seu longo eixo paralelo as fibras musculares.

A captação do sinal foi repetida 3 vezes e foi obtida a média dos 3 para cada situação (antes e após o tratamento) com o intervalo de 2 minutos entre as repetições. Por meio de uma lâmina de parafina com $5 \mathrm{~cm}$ quadrados foram investigadas as situações posturais de repouso mandibular e máxima intercuspidação forçada.

Após a eletromiografia inicial, se deu início à intervenção clínica.

\section{- Confecção das placas miorrelaxantes:}

Inicialmente, os pacientes foram moldados com moldeiras totais metálicas perfuradas individualizadas com cera periférica, e alginato. Os moldes foram vazados com gesso tipo IV e, após a presa, foram recortados e hidratados para montagem em articulador. Posteriormente foi tomado registro do paciente com o arco facial, permitindo a montagem dos modelos maxilares em articulador articulador ${ }^{6}$. Para a montagem dos modelos mandibulares foram tomados registros de mordida em relação cêntrica com Guia de Interferência Mandibular (JIG) com cera número 7 resina acrílica ${ }^{7}$

As placas miorrelaxantes foram confeccionadas sobre estes modelos montados em articulador, segundo a técnica preconizada por Pellissari et al. ${ }^{6}$. Inicialmente, o modelo maxilar recebeu alívio das áreas retentivas e teve toda a extensão da placa delimitada com um lápis. No modelo mandibular foi aplicada vaselina sólida para evitar que a cera com a qual se esculpiu a placa aderisse ao gesso. O passo seguinte consistiu no enceramento da placa de mordida com cera número 7 sobre o modelo superior. Ao término do enceramento a placa foi removida do modelo e incluída numa mufla para prensagem com resina acrílica termopolimerizável incolor. Os excessos foram removidos e a placa polida para não irritar os tecidos moles adjacentes.

Em seguida, a placa foi ajustada na boca de cada voluntário, sendo que, ao final apresentava contatos bilaterais estáveis e simultâneos com os dentes inferiores quando em cêntrica. Em lateralidade o canino inferior deslizava contra uma rampa na placa com total desoclusão e em protrusiva os dentes inferiores tocavam uma rampa anterior desocluindo toda a região posterior da placa. Foram realizados ajustes semanais na placa no intuito de acompanhar o reposicionamento mandibular devido ao relaxamento dos músculos mastigatórios, até a completa estabilização mandibular.

Após o ajuste e instalação da placa, foram dadas instruções aos pacientes em relação à fonética, a qual 
poderia ser mudada nos primeiros dias, pois a placa ocupou o espaço antes preenchido pela língua, a fonética é reestabelecida entre sete a dez dias.

Os voluntários foram orientados a higienizarem as placas com escova e creme dental para evitar alterações na cor do acrílico e acúmulo de placa bacteriana; e a usarem a placa de mordida todos os dias durante o período de sono noturno por 30 dias, e durante este mesmo período, comparecerem à Universidade uma vez por semana para a aplicação de TENS, uma vez que o tratamento proposto consiste nesses dois passos realizados concomitantemente.

\section{- Aplicação de TENS}

Para a aplicação do Transcutaneous Eletrical Nerve Stimulation (TENS), foi utilizado o aparelho TENS Medcir (TENS MT - $104 \mathrm{DX}$ ), modelo 4035, contendo quatro canais de saída.

Inicialmente, as áreas onde os eletrodos foram fixados, região dos músculos masseter e temporal em ambos os lados, foram limpas com álcool $70 \%$ para favorecer a sua fixação. Isso foi feito com o intuito de diminuir a impedância da pele da região de interesse, facilitando a adesão, a captação e a transmissão dos potencias elétricos. Os voluntários foram avaliados e permaneceram sentados e eretos, com os pés apoiados sobre a cadeira odontológica, braços relaxados e costas bem apoiadas.

O aparelho TENS permaneceu no modo normal, com frequência de pulso de $60 \mathrm{~Hz}$ e largura de 300 US. Os eletrodos foram fixados em dois músculos diferentes, que são os responsáveis por esse apertar de dentes tão característicos do bruxismo:

a) músculo masseter (na sua porção mais superficial): foi identificada por palpação, pedindo ao voluntário que execute máxima intercuspidação forçada. No ponto de maior volume, o eletrodo foi fixado;

b) músculo temporal (porção anterior): também foi palpada realizando o mesmo procedimento citado anteriormente, sendo o local de fixação do eletrodo aproximadamente $2,5 \mathrm{~cm}$ anterior e superior à orelha externa.

O aparelho foi ajustado de acordo com a sensibilidade do paciente, evitando contrações musculares e procurando obter-se hipoestesia ou parestesia na região tratada. Cada aplicação foi feita por vinte minutos ${ }^{8}$, uma vez por semana, totalizando quatro semanas.

\section{- Análise de dados:}

Dados descritivos foram apresentados em média desvio padrão e intervalo de confiança. Para análise dos dados descritivos utilizou o teste Shapiro-Wilk (testa normalidade). Para comparação dos dados eletromiográficos utilizou o teste t pareado (paramétrico) e teste Wilcoxon (não paramétrico). Foi adotado $\mathrm{p}<0,05$ utilizando o software Statistical Package for Social Sciences (SPSS), versão 20.0.

Para determinar o tamanho do efeito e o tamanho da amostra foi utilizado o programa GPower 3.1 adotando para tamanho do efeito: efeito baixo $(0-0,4)$, efeito médio $(0,5-$ $0,7)$ e efeito alto $(>0,8)$. Tamanho da amostra foi adotado $\mathrm{p}>0,8$.

\section{RESULTADOS}

Os resultados obtidos nesta pesquisa encontram-se dispostos na Tabela 1.

Observa-se na Tabela 1 a atividade eletromiográfica do músculo masseter e temporal durante o repouso e contração muscular antes (avaliação) e após (reavaliação) o tratamento. Nota-se que, após o tratamento, houve redução significativa $(p=0.028)$ da atividade eletromiográfica para o temporal durante o repouso muscular. De modo geral, observa-se redução da atividade eletromiográfica durante a contração para o masseter e aumento para o temporal, porém sem diferença estatística. $\mathrm{O}$ tamanho do efeito para maioria dos dados foi efeito médio e power foi alto somente para o músculo temporal direito durante o repouso muscular.

Tabela 1. Resultados da análise eletromiográfica dos músculos masseter e temporal durante o repouso e contração muscular no período inicial (Avaliação) e após o tratamento (Reavaliação) com placa miorrelaxante e TENS $(p<0,05)$

\begin{tabular}{|c|c|c|c|c|c|}
\hline Variável & $\begin{array}{c}\text { Avaliação } \\
\pm \sigma \\
\text { IC } 95 \% \\
\end{array}$ & $\begin{array}{c}\text { Reavaliação } \\
\pm \sigma \\
\text { IC } 95 \% \\
\end{array}$ & $p$ & d & Power \\
\hline \multicolumn{6}{|l|}{$\begin{array}{l}\text { Masseter } \\
\text { Repouso }\end{array}$} \\
\hline RMSnD (\%) & $\begin{array}{c}95,95 \pm 4,49 \\
91,79-100,11\end{array}$ & $\begin{array}{c}93,42 \pm 3,93 \\
89,78-97,06\end{array}$ & $0,237^{b}$ & 0,59 & 0,25 \\
\hline RMSnE (\%) & $\begin{array}{c}97,78 \pm 1,78 \\
96,13-99,43 \\
\end{array}$ & $\begin{array}{c}95,62 \pm 1,72 \\
94,03-97,21 \\
\end{array}$ & $0,075^{a}$ & 1,23 & 0,77 \\
\hline \multicolumn{6}{|l|}{$\begin{array}{l}\text { Temporal } \\
\text { Repouso }\end{array}$} \\
\hline RMSnD (\%) & $\begin{array}{c}96,60 \pm 12,01 \\
85,48-107,71\end{array}$ & $\begin{array}{c}82,09 \pm 6,57 \\
76,01-88,17\end{array}$ & $0,028^{b^{\star}}$ & 1,39 & $\underset{\#}{0,84}$ \\
\hline RMSnE (\%) & $\begin{array}{c}96,42 \pm 8,25 \\
88,78-104,06 \\
\end{array}$ & $\begin{array}{c}89,47 \pm 12,51 \\
77,89-101,04 \\
\end{array}$ & $0,128^{b}$ & 0,63 & 0,27 \\
\hline \multicolumn{6}{|l|}{$\begin{array}{l}\text { Masseter } \\
\text { Isométrico }\end{array}$} \\
\hline RMSnD (\%) & $\begin{array}{c}89,91 \pm 6,50 \\
83,90-95,92\end{array}$ & $\begin{array}{c}86,07 \pm 7,07 \\
79,53-92,61\end{array}$ & $0,332^{a}$ & 0,56 & 0,24 \\
\hline RMSnE (\%) & $\begin{array}{c}83,16 \pm 7,66 \\
76,06-90,25\end{array}$ & $\begin{array}{c}84,57 \pm 6,31 \\
78,72-90,41 \\
\end{array}$ & $0,784^{a}$ & 0,19 & 0,07 \\
\hline \multicolumn{6}{|l|}{$\begin{array}{l}\text { Masseter } \\
\text { Isométrico }\end{array}$} \\
\hline FMD & $\begin{array}{l}134,56 \pm 48,46 \\
89,74-179,8\end{array}$ & $\begin{array}{l}120,10 \pm 49,30 \\
74,50-165,69\end{array}$ & $0,359 a$ & 0,29 & 0,10 \\
\hline FME & $\begin{array}{l}155,32 \pm 45,14 \\
113,56-97,07\end{array}$ & $\begin{array}{c}154,36 \pm 22,61 \\
133,45-175,28\end{array}$ & $0,942^{a}$ & 0,02 & 0,05 \\
\hline \multicolumn{6}{|l|}{$\begin{array}{l}\text { Temporal } \\
\text { Isométrico }\end{array}$} \\
\hline RMSnD (\%) & $\begin{array}{l}84,52 \pm 11,99 \\
73,43-95,62\end{array}$ & $\begin{array}{c}87,14 \pm 6,20 \\
81,41-92,88\end{array}$ & $0,306^{a}$ & 0,61 & 0,27 \\
\hline RMSnE (\%) & $\begin{array}{c}91,00 \pm 6,13 \\
85,33-96,68\end{array}$ & $\begin{array}{l}84,03 \pm 11,70 \\
73,21-94,85 \\
\end{array}$ & $0,237 \mathrm{~b}$ & 0,68 & 0,31 \\
\hline \multicolumn{6}{|l|}{$\begin{array}{l}\text { Temporal } \\
\text { Isométrico }\end{array}$} \\
\hline FMD & $\begin{array}{l}172,36 \pm 24,12 \\
150,05-94,68\end{array}$ & $\begin{array}{c}175,89 \pm 39,29 \\
139,54-212,23\end{array}$ & $0,720^{a}$ & 0,10 & 0,05 \\
\hline FME & $\begin{array}{l}65,42 \pm 68,92 \\
1,67-129,17\end{array}$ & $\begin{array}{c}82,01 \pm 57,16 \\
29,14-134,87 \\
\end{array}$ & $0,642^{a}$ & 0,26 & 0,09 \\
\hline
\end{tabular}

Notas: ${ }^{\mathrm{a}}$ Teste t pareado

${ }^{\mathrm{b}}$ Teste Wilcoxon;

$\bar{\chi} \pm \sigma:$ média \pm desvio padrão

IC: intervalo de confiança;

d: Tamanho efeito;

RMSnD: Raiz Quadrada da média normalizada direito

RMSnE: Raiz Quadrada da média normalizada esquerdo;

FMD: frequência mediana direita;

FME: frequência mediana esquerda;

$* \mathrm{p}<0,05$

"power $>80 \%$.

\section{DISCUSSÃO}

Existem patologias que podem acometer os músculos mastigatórios e as articulações temporomandibulares, acarretando o desequilíbrio ou mau funcionamento. Dentre estas, pode-se citar o bruxismo 9 .

$\mathrm{Na}$ presente pesquisa foi observada redução significativa da atividade eletromiográfica do músculo temporal durante o repouso após o tratamento com placa miorrelaxante e TENS. Crosio ${ }^{10}$ relata em seu estudo que a atividade eletromiográfica do músculo temporal durante o repouso após o tratamento com placa interoclusal evoluíram em direção ao equilíbrio cada vez maior da musculatura com a placa, resultado este que assemelha-se ao encontrado nesta pesquisa. Este fato pode ser explicado pela ação fisiológica promovida pela placa que, inserida entre as faces oclusais interrompe a informação proprioceptiva dos mecanoceptores 
localizados no ligamento periodontal permitindo à musculatura mastigatória obter um padrão de atividade balanceada e, consequentemente, manter uma posição mandibular ideal ${ }^{11}$.

Em relação ao TENS, Silveira e Gusmão ${ }^{12}$ relatam que é a terapia que consiste no uso de um aparelho e uma bateria que administra corrente elétrica de baixa voltagem, pulsada, que apresenta uma forma de onda bifásica, simétrica ou assimétrica balanceada com uma semionda quadrada positiva e um pico negativo. Quando ela é aplicada na superfície cutânea através de eletrodos tem como objetivo relaxar os músculos hiperativos e promover o alívio da dor.

Rodrigues et al. ${ }^{13}$ observaram que, em repouso, os indivíduos com DTM apresentam maior atividade mioelétrica dos músculos levantadores da mandíbula, sendo mais evidente na porção anterior do músculo temporal. Quando empregaram a TENS, foi observado alívio da dor com redução simultânea na atividade mioelétrica na porção anterior desse músculo em repouso. Eles postulam que o aumento da amplitude eletromiográfica dos músculos levantadores da mandíbula em repouso deve-se provavelmente a interações sensório-motoras da dor, um sintoma presente em pacientes com DTM e que pode modificar a geração de potenciais de ação e, finalmente, a amplitude da atividade mioelétrica.

Ferreira $^{14}$ relatou em seu estudo sobre o efeito da placa miorrelaxante sobre a atividade elétrica dos músculos temporal anterior e masseter de pacientes com bruxismo, que o músculo temporal esquerdo do Grupo bruxista, nas contrações isométricas, mostrou diferença significativa entre todos os tempos em que foram realizadas as avaliações eletromiográficas. Verificou-se tendência para a diminuição da média da amplitude do sinal eletromiográfico. $\mathrm{O}$ músculo temporal direito apresentou uma tendência de aumento entre as médias dos valores de amplitude do sinal eletromiográfico. $\mathrm{O}$ músculo masseter esquerdo dos voluntários do Grupo bruxista, durante as contrações isométricas, apresentou uma tendência de aumento entre as médias dos valores de amplitude do sinal eletromiográfico, sendo estes resultados compatíveis com os da presente pesquisa. Entretanto, o músculo masseter direito dos voluntários do Grupo bruxista, durante as contrações isométricas, não mostrou diferença significativa entre os tempos T2 (14 dias após) e T3 (21 dias após). Este músculo apresentou uma tendência de aumento das médias dos valores de amplitude do sinal eletromiográfico, diferenciando-se do resultado da presente pesquisa, onde é possível notar redução da atividade eletromiográfica deste músculo, porém sem diferença estatística.

Sobre a confecção da placa, Alencar Júnior et al. ${ }^{15}$ destacaram ser dois os tipos de placas, a placa miorrelaxante convencional rígida e a placa resiliente. Sendo que, a placa miorrelaxante convencional rígida, também denominada de placa de Michigan, é a mais utilizada por causar o menor risco de alterações oclusais definitivas ao paciente, como extrusões, migrações patológicas e mordida aberta anterior e, as placas resilientes, que estariam indicadas principalmente como proteção contra traumatismos nos arcos dentais em algumas modalidades esportivas e nos casos de sinusite crônica que resultasse em grande sensibilidade dentária resultante das forças oclusais fisiológicas.

Jesus ${ }^{16}$ realizou um estudo para avaliar o efeito da placa miorrelaxante sobre o sinal eletromiográfico e a dor em pacientes portadores de DTM e bruxismo. Foram selecionados 30 indivíduos com sinais e sintomas de DTM e bruxismo e 15 indivíduos livres dessas alterações. Os grupos
I e II foram compostos por portadores de DTM e bruxismo sendo que, os participantes do grupo I receberam tratamento com placa miorrelaxante e os do Grupo II com moldeira de silicone (placebo). O grupo III foi composto por indivíduos livres de alterações e não receberam nenhum tipo de intervenção terapêutica. Os indivíduos do grupo I e II utilizaram as placas no período noturno por quatro semanas consecutivas, com ajustes a cada 15 dias. Os resultados mostraram que após 1 mês de terapia com placa estabilizadora ou placa de silicone (placebo) os pacientes portadores de DTM e bruxismo não exibiram melhora do quadro de dor e do sinal eletromiográfico dos músculos masseteres e temporais. Pode-se concluir que o tratamento por 4 semanas com placa de Michigan, não foram suficientes para promover o alívio da dor e alterar a atividade elétrica na amostra estudada.

Entretanto, em relação aos tipos de placas miorelaxantes, Camargos et al. ${ }^{17}$ relataram um caso clínico com pacientes com sinais e sintomas de DTM, onde foram utilizadas placas confeccionadas a partir de uma máquina de prensagem a vácuo, dando a ideia de ser uma placa de um material mais resiliente e no final do seu estudo constatou que o grau de severidade da DTM apresentou-se reduzido ao final da terapia em todos os pacientes, ou seja, mostrando que a placa do tipo resiliente pode ter outras utilidades. Entretanto, de acordo com Montenegro et al. ${ }^{18}$ e Callegari et al. ${ }^{19}$ haverá uma maior redução dos sintomas nas DTMs se a placa for rígida.

Cabral et al. ${ }^{20}$ e Camargos et al. ${ }^{17}$, constataram que a placa miorrelaxante é mais eficiente para o tratamento do bruxismo e os diferentes distúrbios parafuncionais. No estudo realizado por Tsuga et al. ${ }^{21}$ constatou-se que a dor nas ATMs foi a que mais rapidamente respondeu ao tratamento com placa, tendo sua intensidade reduzida em $50 \%$ dos pacientes, com apenas quatro semanas de aplicação. A utilização da placa miorrelaxante promove um significativo alívio da dor, além de reduzir significativamente a amplitude do sinal eletromiográfico nos músculos mastigatórios. ${ }^{20}$

A placa miorrelaxante deve ser utilizada em conjunto com uma série de modalidades, que incluem fisioterapia, farmacoterapia (analgésicos, anti-inflamatórios, relaxantes musculares, antidepressivos), tratamento oclusal (ortodontia, reabilitações, ajuste oclusal); cirúrgico (artroscopia, cirurgia aberta) e psicológico (psicoterapia, psiquiatria) ${ }^{22}$.

\section{CONCLUSÃO}

Considerando-se as limitações do presente estudo, pode-se concluir que o tratamento do bruxismo por meio de placa miorrelaxante rígida e TENS promove redução da atividade eletromiográfica para o músculo temporal durante o repouso muscular, sugerindo que o tratamento proposto proporciona melhora da sintomatologia do bruxismo. No entanto, notou-se a necessidade de intervenção multidisciplinar, visto que o bruxismo está relacionado a fatores locais, sistêmicos, psicológicos e hereditários.

\section{AGRADECIMENTOS}

Ao professor Carlos Antonio da Silva, pelo apoio para a submissão do projeto ao Comitê de Ética em Pesquisa envolvendo seres humanos.

Ao Programa Institucional de Bolsas de Iniciação Científica (PROBIC - UNIFAL-MG), pela apoio financeiro na forma de bolsa de pesquisa de Iniciação Científica.

\section{REFERÊNCIAS}

1. Tan EK, Jankovic J. Treating severe bruxism with botulinum toxin. J Am Dent Assoc. 2000; 131(2):211-6. 
2. Criado L, de La Fuente A, Heredia M, Montero J, Albaladejo A, Criado JM. Electromyographic biofeedback training for reducing muscle pain and tension on masseter and temporal muscles: A pilot study. J Clin Exp Dent. 2016; 8(5):e571-6.

3. Fujisawa M, Kanemura K, Tanabe N, Gohdo Y, Watanabe A, Iizuka $\mathrm{T}$ et al. Determination of daytime clenching events in subjects with and without selfreported clenching. J Oral Rehabil. 2013; 40(10):731-6.

4. Sato M, Iizuka $T$, Watanabe $A$, Iwase $N$, Otsuka $H$, Terada $\mathrm{N}$ et al. Electromyogram biofeedback training for daytime clenching and its effect on sleep bruxism. J Oral Rehabil. 2015; 42(2):83-9.

5. Wang LF, Long H, Deng M, Xu H, Fang J, Fan Y et al Biofeedback treatment for sleep bruxism: a systematic review. Sleep Breath. 2014; 18(2):235-42.

6. Pellissari LP, Lisboa AH, Pellissari MF, Fonseca BR Placas Estabilizadoras: Técnicas de Confecção. J Health. 2010; 4(2):1-9.

7. Fernandes Neto AJ, Das Neves FD, Simamoto Junior PC. Oclusão. São Paulo: Artes Médicas; 2013. 160 p.

8. Rajpurohit B, Khatri SM, Metgud D, Bagewadi A. Effectiveness of transcutaneous electrical nerve stimulation and microcurrent electrical nerve stimulation in bruxism associated with masticatory muscle pain - a comparative study. Indian J Dent Res. 2010; 21(1):104-6.

9. Bataglion C, Palinkas M, Regalo SCH, Siéssere S, Vasconcelos PB, Bueno FL et al. Efeito da placa oclusal resiliente nos músculos masseter e temporal em pacientes com disfunção temporomandibular. Rev Odontol UNESP. 2012; 41(1):27-32.

10. Crosio DM. Eletromiografia dos músculos temporais e masseteres em pacientes com disfunção temporomandibular tratados com placa interoclusal [ dissertação]. Ribeirão Preto: Faculdade de Odontologia de Ribeirão Preto, Universidade de São Paulo; 2010.

11. Landulpho AB, E Silva WA, E Silva FA, Vitti M. Electromyographic evaluation of masseter and anterior temporalis muscles in patiens with temporomandibular disorders following interocclusal appliance treatment. J Oral Rehabil. 2004; 31(2):95-8.

12. Silveira DWS, Gusmão CA. A utilização da estimulação elétrica nervosa transcutânea (TENS) no tratamento da espasticidade - uma revisão bibliográfica. Rev Saúde Com. 2008; 4(1):64-71.

13. Rodrigues D, Siriani AO, Bérzin F. Effect of conventional TENS on pain and electromyographic activity of masticatory muscles in TMD patients. Braz Oral Res. 2004; 18(4):290-5.

14. Ferreira JAND. Efeito da placa estabilizadora do tipo Michigan sobre a atividade elétrica dos músculos temporal anterior e masseter [dissertação]. Piracicaba: Faculdade de Odontologia de Piracicaba, Universidade Estadual de Campinas, Piracicaba; 2001.

15. Alencar Júnior FGP, Aizawa AS, Camparis CM. Placas oclusais e suas indicações no tratamento de pacientes com disfunção craniomandibular (DCM). JBC. 1998; 2(11):56-62.

16. Jesus LA. Efeito da placa miorrelaxante de Michigan sobre a dor e o sinal eletromiográfico em pacientes bruxistas com DTM: um estudo controlado e randomizado [dissertação]. São Paulo: Universidade Nove de Julho; 2008.

17. Camargos GV, Silva MR, Fernandes Neto AJ, Sousa GC, Rodrigues MM. Avaliação das placas oclusais resilientes no controle de pacientes com disfunção temporomandibular (DTM). Horizonte Científico. 2008. 2(1): 1-24.

18. Montenegro FLB, Lascalla NT, Bottino MA, Brunetti RF. Placas de mordida: funções terapêuticas. Rev Paul Odontol. 1984; 6(3):26-42.

19. Callegari A. Terapia com placa oclusal estabilizadora: apresentação de um caso clínico. Rev Paul Odontol. 1999; 21(5):26-31.

20. Cabral LM, Ribeiro AI, Farias ABL, Abrantes DS, Lima LHMA, Ribeiro AI. Placa miorrelaxante no tratamento do bruxismo noturno. Rev ABO Nac. 2008; 15(6 Suppl 1):85.

21. Tsuga K, Akagawa Y, Sakaguchi R, Tsuru H. A shortterm evaluation of the effectiveness of stabilization-type occlusal splint therapy or specifc syptons of tempoomandibular joint dysfunction syndrome. J Prosthet Dent. 1989; 61(5): 610-3.

22. Conti P. Entrevista: Paulo Conti. Rev Dent Press Ortodon Ortop Facial. 2006; 11(2):18-28.

\section{CONFLITO DE INTERESSES}

Os autores declaram não haver conflitos de interesse.

\section{AUTOR PARA CORRESPONDÊNCIA}

\section{Marcela Filié Haddad}

marcela.haddad@unifal-mg.edu.br

Submetido em 21/06/2017 Aceito em 13/07/2017 